\title{
Original
}

\section{Estado nutricional de la población menor de 5 años en la consulta de crecimiento y desarrollo del distrito San Rafael de Alajuela en el año 2002.}

Manrique Leal - Mateos ', Javier Ruiz- Castro ${ }^{2}$, Ricardo Salazar- Solís ${ }^{3}$

\author{
Resu m e n
}

Médico Director, Área Rectora de Salud de Alajuela 2.

2. Médico Asistente Encargado de Vigilancia Epidemiológica, Área Rectora de Salud de Alajuela 2.

3. Médico Asistente Encargado de Regulación de los Servicios de Salud, Área Rectora de Salud de Alajuela 2.

Abreviaturas: C.C.S.S: Caja Costarricense del Seguro Social; EBAIS, Equipo Básico de Atención Integral en Salud; NCHS, National Center for Health Statistics.

Correspondencia: Dr. Manrique Leal Mateos , Apartado 55 Centro Comercial San José 2000, Fax: 296-340I.

ISSN 0001-6002/2004/46/2/72-77 Acta Médica Costarricense,(C2004 Colegio de Médicos y Cirujanos
Justificación y objetivos: Las encuestas nutricionales realizadas en Costa Rica permiten tener una idea general del estado nutricional de la población costarricense. Sin embargo, no facilitan información precisa de lo que sucede en las diferentes áreas de salud del país, ni del desempeño de los programas preventivos.

El presente estudio pretende determinar el estado nutricional de la población menor de 5 años que asistió a la consulta de crecimiento y desarrollo en el distrito de San Rafael de Alajuela, durante el periodo 2002, y comparar la situación nutricional de la población en estudio, con los resultados de obtenidos en la última Encuesta Nacional de Nutrición, de 1996.

Métodos: Se trabajó con la población de niños(as) menores de 5 años que asistió a la consulta de crecimiento y desarrollo del primer nivel de atención en salud. A partir de la muestra obtenida, se caracterizó a la población, según los indicadores antropométricos peso/edad, talla/ edad y peso/talla.

Resultados: Se revisó un total de 275 expedientes. La mediana de edad fue de 18 meses y la edad más frecuente de consulta fue de 12 meses. Según el indicador peso/edad, 33 (12\%) niños(as) presentaron algún grado de desnutrición y 9 (3.3\%), sobrepeso.

El número de niños(as) con problemas de desnutrición y sobrepeso fue semejante en ambos sexos; igualmente, la cantidad de pacientes con problemas de desnutrición y sobrepeso en el grupo de niños(as) menores de 1 año, fue semejante al de 1 a 4 años.

Conclusión: Al comparar los resultados obtenidos con la última encuesta nacional de nutrición, se determinó que los(as) niños(as) que asisten a la consulta de crecimiento y desarrollo, presentaron un mejor estado nutricional que el de la población nacional.

Descriptores: estado nutricional, crecimiento y desarrollo, indicadores antropométricos.

Recibido: 1 de julio de 2003

Aceptado: 20 enero de 2004

La nutrición representa un factor indispensable para que se desarrollen todas las potencialidades físicas, biológicas e intelectuales del ser humano. Sus necesidades varían de acuerdo con las diferencias genéticas y metabólicas de cada individuo ${ }^{1-3}$. 
El crecimiento y desarrollo asociado al estado nutricional constituyen uno de los índices más sensibles y útiles para determinar el nivel de desarrollo y la calidad de vida de la población, debido a que involucran una perspectiva dinámica y evolutiva del ser humano ${ }^{1.2}$.

La malnutrición es reconocida como una de las principales causas de morbilidad y mortalidad, sobre todo en los países en vías de desarrollo ${ }^{3}$. Los trastornos nutricionales están relacionados con alteración en los patrones de crecimiento y desarrollo de los niños, específicamente en lo que respecta a la baja talla y la dificultad para progresar ${ }^{3.4}$.

La mayoría de las encuestas nutricionales hechas en Latinoamérica muestran que la desnutrición es un proceso crónico más que agudo, que se refleja en un crecimiento lineal. Además, afirman que su prevalencia es mayor en las zonas rurales que en las urbanas. En varios países de América Central, como El Salvador, Honduras y Nicaragua, la prevalencia de desnutrición en zonas áreas rurales llega a ser casi el doble que en las urbanas ${ }^{5}$.

Son pocos los países con suficientes datos seculares sobre el estado nutricional de su población, lo que no permite un análisis preciso de la tendencia que mantiene tal problema ${ }^{5,6}$.

En Costa Rica se han realizado cinco encuestas para conocer el estado nutricional de la población. Sin embargo, es importante destacar que hasta 1996 no se contaba con datos precisos que permitieran conocer la situación nutricional de los menores de seis años ${ }^{1}$.

La Encuesta Nacional de Nutrición de 1996 reveló que de acuerdo con el indicador peso/edad, el $22.4 \%$ de los preescolares presentó algún grado de desnutrición o sobrepeso. Asimismo, mencionó que la población preescolar de Costa Rica acusó una mejoría, al disminuir la prevalencia de la desnutrición moderada y grave, así como el retardo en talla ${ }^{1}$.

Se observó también un predominio de la desnutrición crónica en relación con la aguda. Las zonas rurales del país presentaron los mayores porcentajes de desnutrición.

El sobrepeso en este grupo poblacional aumentó en comparación con los años anteriores ${ }^{1}$.

El estudio pretende determinar el estado nutricional de la población menor de 5 años que asistió a la consulta de crecimiento y desarrollo en el distrito de San Rafael de Alajuela, durante 2002 y comparar la situación nutricional de la población en estudio con los resultados obtenidos en la última Encuesta Nacional de Nutrición, de 1996.

\section{Materiales y métodos}

La población de estudio corresponde a los (as) niños(as) menores de 5 años de edad, que consultaron en los cuatro EBAIS del distrito de San Rafael de Alajuela, durante 2002.
El marco muestral considerado lo formaban 1024 niño$\mathrm{s}(\mathrm{as})$ incluidos en el registro estadístico de la Consulta de Crecimiento y Desarrollo de los cuatro EBAIS. Para calcular el tamaño de la muestra se utilizó una prevalencia esperada del 22.4\% según la Encuesta Nacional de Nutrición de 1996 1. Se estimó un error esperado del $5 \%$ y un intervalo de confianza del 95\%, para lo cual se utilizó el sistema Statcalc del programa EpiInfo 2000.

Con estos (as) 1024 niños(as), se obtuvo una muestra de 275 expedientes clínicos, mediante un muestreo aleatorio y sistemático. El tamaño de la muestra fue incrementado en un $10 \%$, para mantener su representatividad y aumentar el grado de confianza.

Se identificó a cada individuo por su número de expediente. La recolección de los datos se realizó mediante una hoja precodificada que incluyó las variables: edad, sexo, peso y talla. Posteriormente, los resultados fueron ploteados en gráficas de crecimiento somático, a partir de la relación de los indicadores antropométricos talla/edad, peso/edad, y peso/talla, para caracterizar a cada individuo de acuerdo con el tipo y grado de malnutrición.

Las tablas utilizadas para la clasificación de los pacientes se basaron en el patrón de referencia del National Center for Health Statsitics, empleado en las Normas de Atención para el manejo integral del niño con desnutrición ${ }^{7}$. Este define como sobrepeso aquellos niños con más de +2 desviaciones estándar; normal, los que se encuentran entre +2 y -0.9 desviaciones estándar; leve, los que se ubican entre -1 y -1.9; moderada, entre - 2 y -2.9 y desnutrición grave menor o igual a -3 desviaciones estándar.

Con base en el mismo patrón de referencia, se definió como alto para edad aquellos niños con más de +2 desviaciones estándar; normal, los que se encuentran entre +2 y -0.9 desviaciones estándar; baja talla, entre -1 y -1.9 y retardo en talla menor o igual a -2 desviaciones estándar.

Para todos los casos se tomó la información de la última consulta de 2002.

Los criterios de exclusión fueron los siguientes:

1. Cualquier niño(a) que durante el periodo de estudio no se encontrara dentro del rango de edad establecido.

2. Cualquier niño(a) que no perteneciera al distrito de San Rafael de Alajuela.

3. Aquellos cuya información estuviera ausente o incompleta en el expediente clínico.

4. Cualquier niño (a) que presentara alguna patología aguda que pudiera afectar los resultados obtenidos de las mediciones antropométricas al momento de la consulta. Para los dos últimos casos, se utilizó como registro la consulta previa a esta, realizada el mismo año. 
5. Se excluyó por completo a aquellos(as) niños(as) que presentaron patologías crónicas que afectaran su estado nutricional.

El registro básico se introdujo en una base de datos informatizada, utilizando el programa EpiInfo 2000. Para el análisis se utilizaron distribuciones de frecuencias, con valores absolutos y porcentuales, medidas de tendencia central y de dispersión. La relación entre variables cualitativas se realizó mediante la prueba estadística de Ji cuadrada $\left(\mathrm{X}^{2}\right)$. El nivel de significancia fue fijado en $p<0.05$. Los resultados se presentaron en tablas y gráficos elaborados en el programa Excel 2000.

\section{Resultados}

La población del distrito San Rafael de Alajuela cuenta con 19.162 habitantes, de acuerdo con el último censo realizado por el Instituto Nacional de Estadística y Censo, en 2000 8. Los niños(as) menores de 5 años de edad corresponden a 1823 (9.5\% del total).

De los 275 expedientes seleccionados se encontró que 97 (35.3\%) fueron menores de un año y 178 (64.7\%), de entre 1 y 4 años.

La mediana de edad fue de 18 meses y la edad más frecuente de consulta fue de 12 meses.

En cuanto al sexo, del total de niños (as) estudiados (as), $109(39.6 \%)$ pertenecieron al sexo femenino y $166(60.4 \%)$ al masculino.

Se observó, según el indicador peso/edad, que del total de la muestra, 233 (84.7\%) presentaron un estado nutricional normal y 33 (12\%), algún grado de desnutrición (Figura1).

Para el indicador peso/talla, se encontró que 40 (14.6\%) manifestaron problemas de desnutrición. De estos, 34 (12.4\%) presentaron desnutrición leve, 5 (1.8\%) moderada y $1(0.4 \%)$ grave. El número de niños(as) obesos (as) fue de $24(8.7 \%)$.

Se evidenció que del total de niños (as) estudiados (as), según el indicador talla/ edad, 58 (21.1\%) presentaron algún déficit en talla. De estos, se encontró que 56 (20.4\%) presentaron baja talla y $2(0.7 \%)$, retardo en talla. Además, $2(0.7 \%)$ niños resultaron altos para la edad.

Los resultados revelaron que de los 166 hombres estudiados, $139(83.7 \%)$ presentaron un estado nutricional normal y $20(12 \%)$, algún problema de desnutrición de acuerdo con el indicador peso /edad (Figura 2).

A partir del indicador peso /talla, se observó que del total de hombres, 20 (12\%) presentaron problemas de desnutrición. De ellos, 18 (10.8\%) correspondieron a desnutrición leve, $1(0.6 \%)$ a desnutrición moderada y $1(0.6 \%)$ a

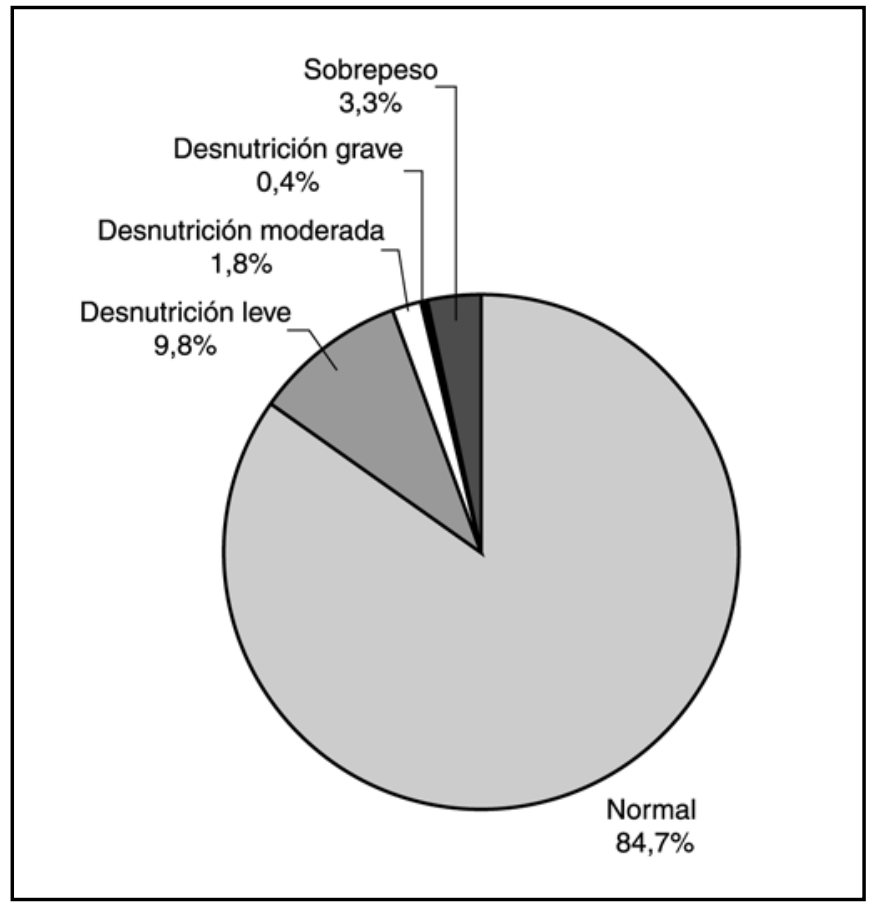

Figura 1. Estado nutricional de la población menor de 5 años, según peso para edad, Consulta de crecimiento y desarrollo, Distrito San Rafael de Alajuela, 2002.(Cifras relativas).

Fuente: Expedientes Clínicos de la Consulta de Crecimiento y Desarrollo de los cuatro EBAIS, Distrito San Rafael de Alajuela, 2002.

desnutrición grave. El número de niños con obesidad fue de $17(10.2 \%)$.

Se determinó, según el indicador talla/edad, que 31 (18.7\%) hombres presentaron déficit en su talla, 29 (17.5\%) de ellos, baja talla y $2(1.2 \%)$, retardo en talla.

Se encontró que de las 109 mujeres estudiadas, 94 (86.2\%) presentaron un estado nutricional normal y 13 (12\%), algún grado de desnutrición, de acuerdo con el indicador peso/ edad (Figura 2).

Se determinó, según el indicador peso/ talla, que de las mujeres estudiadas, 20 (18.4\%) presentaron algún grado de desnutrición. Se encontró que 16 (14.7\%) manifestaron desnutrición leve y 4 (3.7\%), moderada. El número de niñas con problemas de obesidad fue de 7 (6.4\%).

Con base en el indicador talla/edad, se comprobó que 27 $(24.8 \%)$ de las mujeres presentaron déficit en su talla (baja talla) y $2(1.8 \%)$ resultaron altas para la edad.

De acuerdo con el indicador peso/edad, no existe una relación estadísticamente significativa entre el sexo de los pacientes y la desnutrición ( $\mathrm{p}=0.9171)$; esto también fue observado al relacionar el sexo con el sobrepeso $(\mathrm{p}=0.2755)$.

Se determinó que de los 97 niños menores de un año, $87(89.7 \%)$ presentaron un estado nutricional normal y 7 (7.2\%), algún grado de desnutrición, de acuerdo con el indicador peso/edad (Figura 3). 


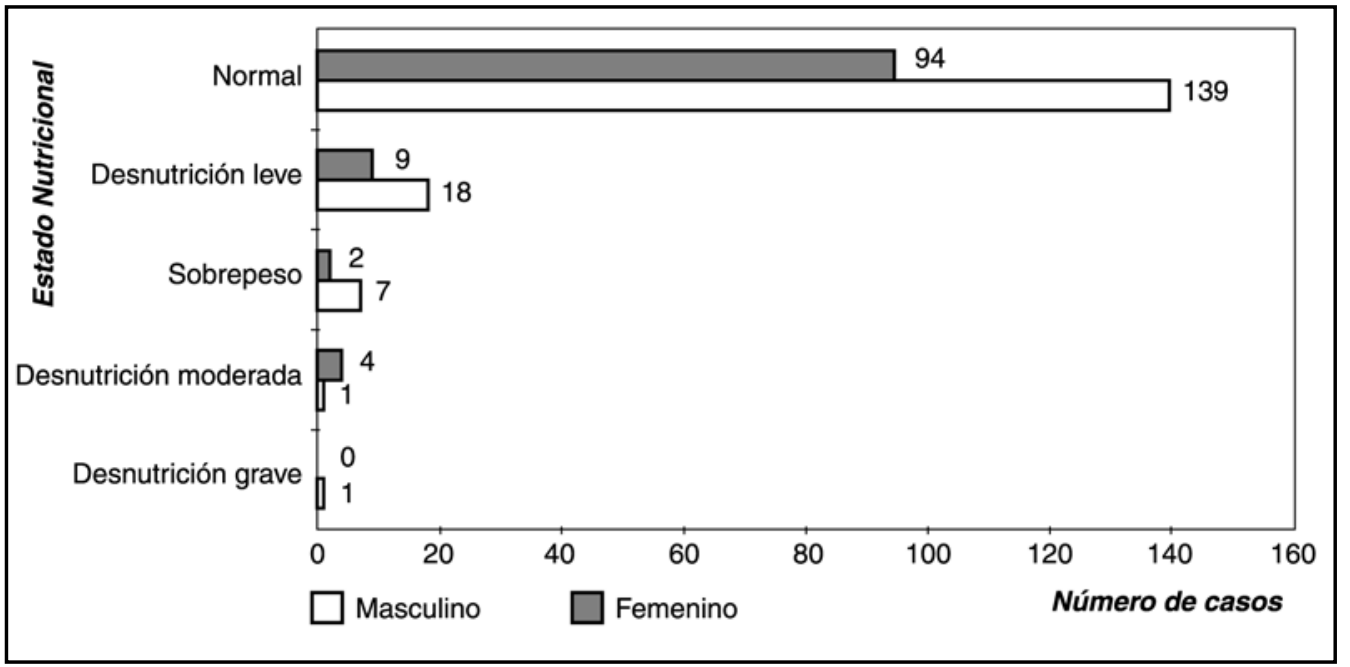

Figura 2. Estado nutricional de la población menor de 5 años, por sexo según peso para edad, Consulta de crecimiento y desarrollo, Distrito San Rafael de Alajuela, 2002.(Cifras absolutas).

Fuente: Expedientes Clínicos de la Consulta de Crecimiento y Desarrollo de los cuatro EBAIS, Distrito San Rafael de Alajuela, 2002

Del total de niños para este grupo etáreo, se observó, según el indicador peso/talla que $8(8.2 \%)$ presentaron algún tipo de desnutrición. De estos, $6(6.2 \%)$ resultaron con desnutrición leve, 1 (1\%) con desnutrición moderada y $1(1 \%)$ con desnutrición grave. Además, se presentaron 10 casos (10.3\%) de niños con problemas de sobrepeso.

Según el indicador talla/edad, se encontró que 19 (19.6\%) presentaron algún déficit en su talla (Cuadro 1).

Se evidenció que de los 178 niños entre 1 y 4 años de edad, $146(82 \%)$ mostraron un estado nutricional normal y $26(14.6 \%)$ resultaron con problemas de desnutrición, el indicador peso/edad (Gráfico 3).

Para el indicador peso/talla se estableció que 32 (17.9\%) de los niños presentaron problemas de desnutrición, de los cuales $28(15.7 \%)$ manifestaron desnutrición leve y 4 (2.2\%), desnutrición moderada. El número de niños con sobrepeso es de $14(7.9 \%)$.

De acuerdo con el indicador talla/edad, se encontró que de los 178 niños en este rango de edad, 39 (21.9\%) presentaron algún déficit en su talla (Cuadro 1).

Si bien, con base en el indicador peso/edad se observó un mayor número de niños(as) con desnutrición en el grupo de 1 a 4 años, esta diferencia no fue estadísticamente significativa al relacionarla con el grupo de menores de 1 año $(\mathrm{p}=0.06)$. Tampoco se observó asociación entre estos grupos etáreos y el sobrepeso $(\mathrm{p}=0.80)$.

\section{D is cus ión}

El estado nutricional de los preescolares se determinó mediante el indicador peso/edad, el cual refleja la historia nutricional actual y pasada, sin distinguir entre desnutrición aguda o crónica. Este Indicador es sensible, pero poco específico, por lo que se recomienda utilizarlo para niños menores de 6 años. Además, es el primero en modificarse cuando se presentan deficiencias nutricionales ${ }^{1,9}$.

El indicador peso/talla es el más específico para diagnosticar la desnutrición actual o aguda, ya que es independiente de la edad hasta la adolescencia. Es, además, más sensible en niños con talla alta donde el indicador peso/edad ocasiona falsos negativos.

El indicador talla/edad refleja el estado nutricional pasado, por lo tanto es el utilizado para valorar la desnutrición crónica. Sin embargo, es menos sensible y se modifica más tardíamente.

Para categorizar de manera adecuada al niño, se debe evaluar, idealmente, mediante una correlación de las tres ${ }^{9}$.

Con base en el indicador peso/edad, el grado de desnutrición predominante en la población estudiada fue el leve, lo anterior concuerda con los resultados obtenidos a nivel nacional y en la zona urbana, en la última encuesta de nutrición ${ }^{1}$.

El tipo de desnutrición predominante fue la crónica, al presentarse un mayor porcentaje de casos, según el indicador talla/edad. Los resultados obtenidos coinciden con la Encuesta Nacional de Nutrición realizada en $1996{ }^{1}$.

\section{Cuadro 1.}

Número de casos por grupo etáreo según

Talla para Edad, Consulta Crecimiento y Desarrollo, Distrito San Rafael de Alajuela, 2002.

\begin{tabular}{|l|l|}
\hline & \multicolumn{2}{|c|}{ Rango de edad } \\
\cline { 2 - 2 } Talla para edad & Menores de 1 año De 1 a 4 años \\
\hline
\end{tabular}

\begin{tabular}{lcc|}
\hline Normal & 77 & 138 \\
Baja & 18 & 38 \\
Retardo & 1 & 1 \\
Alto & 1 & 1 \\
\hline Total & 97 & 178 \\
\hline
\end{tabular}

Fuente: Expedientes Clínicos Consulta de Crecimiento y DesarroIlo de los cuatro EBAIS, Distrito San Rafael de Alajuela, 2002. 


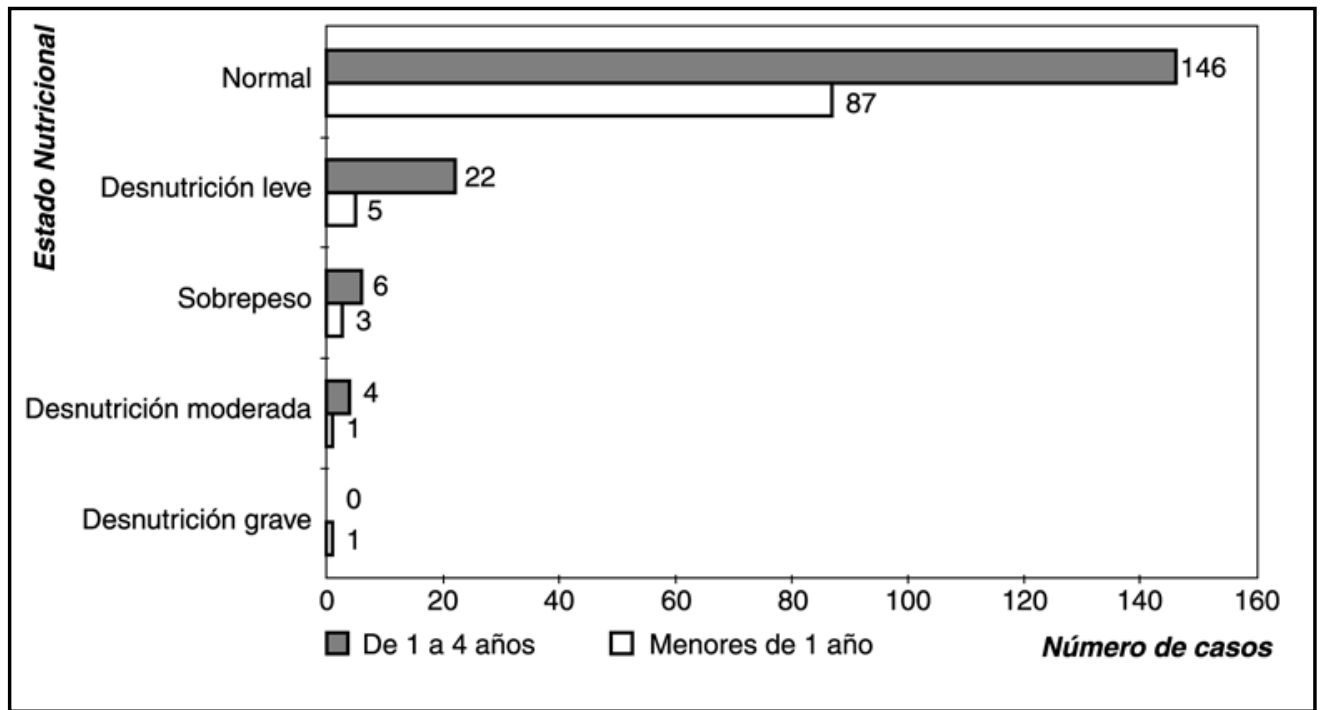

Figura 3. Estado nutricional de la población menor de 5 años, por grupo etáreo según peso para edad, Consulta de crecimiento y desarrollo, Distrito San Rafael de Alajuela, 2002.(Cifras absolutas).

Fuente: Expedientes Clínicos de la Consulta de Crecimiento y Desarrollo de los cuatro EBAIS, Distrito San Rafael de Alajuela, 2002

No obstante, el porcentaje de niños con algún grado de desnutrición es menor que el obtenido a nivel nacional.

El predominio de la desnutrición crónica en la población menor de 5 años, puede reflejar que el personal de salud considera que la baja talla es normal en el país, debido a factores genéticos, y da una menor importancia a factores ambientales, lo que hace que los niños no sean beneficiarios de los programas preventivos para mejorar su estado nutricional ${ }^{6,10}$.

El sobrepeso representó el 3,3\% de la muestra, con un menor porcentaje que el reportado en la Encuesta Nacional de Nutrición ${ }^{1}$.

El número de pacientes con desnutrición, según el indicador peso/edad, fue semejante en ambos sexos. Se puede concluir que esta variable no fue significativa en el desarrollo de este trastorno nutricional; estos datos difieren de los encontrados en la Encuesta Nacional de Nutrición, donde se evidenció un mayor deterioro nutricional en el sexo masculino.

Se pudo determinar una mayor presencia de sobrepeso en los varones que en las mujeres, pero con una diferencia no estadísticamente significativa. En la Encuesta Nacional de Nutrición se evidenciaron porcentajes similares de sobrepeso en ambos sexos.

Al comparar los resultados obtenidos para ambos grupos de edad, se documentó que el porcentaje de niños con algún grado de desnutrición fue mayor en la población de 1 a 4 años, pero esta diferencia tampoco fue estadísticamente significativa. Aún así, esto puede obedecer al factor protector de la lactancia materna durante el primer año de vida, la mayor preocupación de los padres en esta etapa de desarrollo o un mayor contacto con el personal médico y los servicios de salud, que hacen que los trastornos nutricionales se diagnostiquen tempranamente y sean corregidos de forma precoz ${ }^{11-15}$.

En términos generales, la población que asiste a la consulta de Crecimiento y Desarrollo del primer nivel de atención de la CCSS, en el distrito de San Rafael de Alajuela, presenta un mejor estado nutricional que la población nacional.

Lo anterior puede resultar de los esfuerzos implementados en los últimos años por las autoridades de salud, en cuanto a con los programas preventivos del primer nivel de atención.

La educación de los padres de familia, sobre la importancia del control nutricional, resulta esencial para el buen desarrollo de los niños. Aunque la preocupación de estos es mayor en el primer año de vida, se deben dirigir esfuerzos para fortalecer la educación de los padres y garantizar un control nutricional continuo, periódico $\mathrm{y}$ adecuado, hasta el inicio de la adolescencia ${ }^{7,16,17}$.

\section{A bstract}

Nutritional surveys carried out in Costa Rica provide an overview of the general nutritional status of our population. However, they don't provide accurate information as to what happens in different health areas in the country or data about the performance of prevention programs.

The present study, therefore, is aimed at determining the nutritional status of the population under 5 years of age who visited the primary care centers in the district of San Rafael, province of Alajuela in 2002, and to comparing the nutritional status of the target population with the results from the last national nutritional survey from 1996.

The target population consisted of children under 5 years old who visited the primary care centers. Based on the sample, a population characterization was carried out according to anthropometrical indicators of weight/age, size/age, and weight/size.

A total of 275 records were analyzed. The mean age was 18 months and the most frequent age was 12 months. According to the weight/age indicator, $33(12 \%)$ of the children showed certain degree of malnutrition and $9(3.3 \%)$ had overweight. The percentage of children suffering from malnutrition or overweight was similar for both genders and ages. 
After comparing results from the last national nutritional survey, it was determined that children who visited the primary care centers showed a better nutritional status than the national population.

\section{Referencias}

1. Ministerio de Salud. Encuesta Nacional de Nutrición, Fascículo Antropometría. San José, Costa Rica: Ministerio de Salud,1996.

2. Murillo S. Estado nutricional de la niñez y la adolescencia en Costa Rica. En: UNICEF. Nuestro derecho a la nutrición y salud en Costa Rica. Costa Rica: UNICEF, 1999: 46 - 52.

3. Guendel L. El marco jurídico internacional sobre los derechos de la niñez y la nutrición. En: UNICEF. Nuestro derecho a la nutrición y salud en Costa Rica. Costa Rica: UNICEF, 1999: 4-15.

4. Jiménez C. Falla para progresar. Enfoque del pediatra general. En: Madrigal G, ed. Manual de diagnóstico y terapéutica en pediatría. Costa Rica : Editorial de la Universidad de Costa Rica, 1999: 624-629.

5. Organización Panamericana de la Salud. Enfermedades y daños a la salud. En: Organización Panamericana de la Salud, ed. La salud en las Américas. Washington: OPS,1998: 161-168.

6. Delgado H. Uso de patrones antropométricos en la región centroamericana: Aciertos y desaciertos. En: Cambronero M, Monge R, Murillo S, eds. Taller centroamericano de antropometría en la población menor de 19 años. San José, Costa Rica: INCIENSA, 1999: 6-13.

7. Morice A. Situación de las enfermedades crónicas no transmisibles en Costa Rica. San José: INCIENSA, 1998.

8. Instituto Nacional de Estadística y Censo. Cálculos de población por provincia, cantón y distrito al $1^{\circ}$ de enero al $1^{\circ}$ de julio 2000-2002, San José: INEC, 2001
9. Tacsan L. La vigilancia alimentaria y nutricional en Costa Rica. En: UNICEF ed. Nuestro derecho a la nutrición y salud en Costa Rica. Costa Rica: UNICEF, 1999: 16-23.

10. Beherman R, Kliegman R. Nelson Tratado de pediatría. México: Mac Graw - Hill, 2001.

11. Betrán I, De Onís M, Lauer J, Villar J. Ecological study of effect of breast feeding on infant mortality in Latin America . BMJ 2001; 323:303-306.

12. Buñuel J, Vila C, Puig M, Díez S, Corrral A, Pérez M . Influencia del tipo de lactancia y otros factores sobre la incidencia de infecciones del tracto respiratorio en lactantes controlados en un centro de atención primaria. Aten Primaria 2002; 29: 268 - 277.

13. Wright A, Bauer M, Naylor A, Sutcliffe E, Clark L. Increasing breast feeding rates to reduce infant illness at the community level. Pediatrics 1998; 101: 837 - 844.

14. H Rubin, Levanthal J, Krasilinikoff P, Kuo H, Jekel J, Weile B, et al. Relationship between infant feeding and infectious illnes: A prospective study of infants during the first year of life. Pediatrics 1990; 85: 464 -471 .

15. Kramer M, Chalmers B, Hodnett E, Sevkovskaya Z, Dzikovich I, Shapiro S, et al. Promotion of breast feeding intervention trial (PROBIT) : A randomized trial in the Republic of Belarus. JAMA 2001; 285. 413 -420 .

16. Esquivel M, Romero J, Berdasco A, Gutiérrez J, Jiménez J, Posada E, et al. Estado nutricional de preescolares de Ciudad de la Habana entre 1972 -1993. Rev Panam Salud Pública 1997; 1: 349 - 353.

17. Ministerio de Salud. Normas de atención para el manejo integral del niño(a) con desnutrición en los diferentes niveles de atención. San José , Costa Rica: Ministerio de Salud, 1999. 